\title{
Sustainable Advertising based Optimal Media Selection for Segmented Market
}

\author{
P. C. Jha \\ Department of Operational \\ Research, Faculty of \\ Mathematical Sciences, \\ University of Delhi, India
}

\author{
Remica Aggarwal \\ School of Business, University of \\ Petroleum \& Energy Studies, \\ Dehradun, India
}

\author{
S. P. Singh \\ Department of Management \\ Studies, IIT Delhi, India
}

\author{
P. K. Kapur \\ Amity Center for Interdisciplinary Research \\ Amity University, Noida, India
}

\begin{abstract}
Promotion or advertising is an important form of marketing and an imperative phenomenon from time immortal to gain success over the competitors. It is gradually changing to sustainable or green marketing which encompasses various possible aspects ranging from ecological to economic to sustainability. The case in the present paper consists of introducing a new FMCG product in the potential market . The multiple objectives include maximizing the uncertain advertising media reach for different media options subject to the constraints associated with the different processes of advertising and advertising budget constraints. Sustainable media options chosen are recyclable paper print media and website media. The problem is formulated as the multiple conflicting objectives optimization problem which can be solved using Non pre-emptive goal programming approach.
\end{abstract}

\section{Keywords}

Media allocation, green media selection, multi-criteria decision making, goal programming approach

\section{INTRODUCTION}

In addition to green supply chain management and new product innovation and diffusion which have become popular research areas past few years, eco marketing or ecological marketing or green marketing is started becoming known to people [1-4]. Companies are increasingly adopting the carbon footprint labels [5]. This not only make them competitive enough to stay ahead but also create a status quo for them in the market and hence add a goodwill. However, meeting all the three phenomena together is troublesome for many and conducting a research on this hybrid concept represents a comparably new trend and need to be explored further. It is important that marketers integrate green marketing strategies carefully into the company strategic plan. When it comes to awareness regarding eco-friendly products, consumers mostly trust eco-labels/eco-ratings followed by news reports, recommendation by known people and lastly to paid advertisements by the manufactures, while also looking for opinions and information posted by other consumers online.

Present research work target FMCG manufacturing industry of India . The Indian FMCG sector is the fourth largest in the Indian economy having a market size of approximately $\$ 13.1$ billion. This industry primarily manufacture consumer packaged goods. The potential market of such products primarily includes middle class and the rural segments of the Indian population and give brand makers the excellent opportunity to convert them to branded products. It includes groceries and drinks, personal care, plastic goods, paper and stationery and household products etc. However, only personal care products are taken into consideration for this study.

Different queries arise in the mind of researcher in different aspects considering sustainability marketing or green marketing or advertising in an appropriate green media [5,7]. For example, some of the obvious questions related to alignment of sustainability marketing strategies with the organization's objectives could be

- How to align the marketing strategy to a corporate sustainability objective?

- Does the company have a stated environmental policy?

- Is this policy clearly articulated within the organization?

- Has the communication strategy kept pace with the business's sustainable ambition?

Similarly, considering the media sustainability optimization, the obvious questions could be

- How to select the media to ensure minimal environmental impact

- Is the organization advertising and marketing environment friendly products or are promoting itself as an environmental conscious organization?

Researchers have basically conducted empirical research to get answer to the above questions. In this research paper, a mathematical approach has been presented to study the green media selection and allocation problems. The aim is to find the number of advertisements that could be placed in different media options so as to maximize the uncertain advertising reach and effectiveness amongst the potential adopters of a new FMCG product. Here the media that have been considered are the recyclable print media options and internet websites. Various media are given different weightage so as to capture maximum effectiveness. The cost aspects for the different pages of the newspapers and different time slots of websites have been given due consideration. The research paper takes in to account uncertainty associated with the advertising reach. Non pre-emptive goal programming approach has been applied to provide a compromise solution 
to the problem.

The research work is organized as follows. Section 2 briefly presents the literature review. Section 3 presents the problem definition. Section 4 defines the mathematical model formulation. Section 5 describes the non pre -emptive goal programming methodology. Section 6 presents the conclusions and future directions .

\section{LITERATURE SURVEY}

Media in the marketing communication literature is often associated with traditional mass media. Different advertising media generates different communication effects even when same advertisements are used towards the same target audience $[2,4,3,6]$. This clearly means that if media is chosen intelligently, it can reap rewards for the marketers. However, choice of the media depends on various factors such as target consumer market characteristics, perception and purchase behavior and type of product to be marketed . A firm should select the media through which its products can be best advertised keeping in mind the amount of resources (financial and human resources) available.

Advertising and media investments range from the simple use of newspaper advertisements to complex global web advertising and the number of alternative media to reach out to consumers. Regardless of the media type used to communicate to an audience, advertising can be used to market an offer, to provide knowledge about certain brands or to educate people about important issues in society. As each medium has its advantages and disadvantages, choice of media as mentioned before is guided by various factors such as extent of exposure to the advertisers' message in a given time period (i.e. frequency ), number of target audiences who are expected to exposed to the advertisement (i.e. advertising reach), its impact and costs but also in terms of how each of the selected media from the media mix complements each other, i.e. integrated marketing communication. Advertising media planners uses each of the aforementioned media in varying degrees (i.e. assigning different weights to different media) to reach large audiences while minimizing costs. These weights can be decided by the management based on their experience or through past data collection. The optimal media planning for segmented market for single as well as multiple products etc. has been proposed by Jha et al. [10,11].

Energy consumption and power generation are the major key drivers for online and broadcast media and therefore it should be kept in mind while assessing the green environmental impact of media $[8,9,16,14]$. Consequently, consumers also have beliefs about the use of different media regarding its impact on the green environment. [16,17] explored the various scenarios of emerging media trends and customer response and perception towards various media options while doing green marketing. Generally, they feel that paper based media is more harmful than electronic media and therefore the context of placing an ad in a paper based medium may also be perceived as more ecologically harmful than when placed through an electronic medium and vice versa.

Three different forms of media options based on direct mail, city bus and internet advertising was proposed by $[8,14]$ with their pros and cons and it was found that internet media was considered the best amongst the given media options. According to the study, major criteria which affects the choice of green media for advertising includes the medium's measurability, reach or frequency or impact, product type, target group, the communication objective, medium's green characteristics, medium's communicating characteristics, consumers' media attitudes, availability of media space and budget or cost related criteria. Further the media selection among marketing managers include green organizational ideology, green personal beliefs and model use . Company manager's point of view and their perception have been studied by while using green media [14-16]. In a recent study, [13] analyze the interviews with parents and their children to define the ways in which they act sustainably towards media devices and content available at home. Its main contribution concerns provide insights in to the implications of the current sustainable media use of families with young children for future generations .

A linear programming model proposed by De Kluyver [3] makes use of hard and soft constraints in media selection. [4] discussed the best possible combination of placements of commercial with the goal of the highest rating subject to advertisement budget constraint. [18] has presented a mixed integer goal programming model for industrial and consumer market for high technology products . A pre-emptive goal programming model to find the optimal number of advertisements that can placed in different media under the advertising budget constraint is proposed by [12]. The above models have not included the practical aspect of segmentation. A media planning model that assists a firm in determining the optimal media mix for a product advertised in a segmented market. The model determines the number of advertisements to be placed in different segment specific media as well as mass media $[10,11]$.

\section{PROBLEM DEFINITION}

The product is a new cosmetic product which needs to be advertised through recyclable print media and website media to the target market segments which are segmented based on criteria such as age and income levels. The objectives are multiple corresponding to maximization of advertising reach associated with various media options considered subject to the advertising cost budget constraint along with the number of advertisements to be inserted in different media etc.

\section{MODEL FORMULATION}

\subsection{Assumptions of the model}

$>$ Market is segmented based on the criteria such as age and income

$>$ Multiple segments are considered within each demand centre or potential demand market

$>$ There is a single manufacturing plant manufacturing a product and delivering it to multiple demand centres

$>$ There would be an advertising cost function in terms of units manufactured which will be something like a per unit advertising cost associated with the number of units manufactured.

\subsection{Notations}

$s \quad: 1,2, \ldots . . \mathrm{S}$ segments

$m: 1,2, \ldots \mathrm{M}$ medium of advertising

$d$ : demand centres $(d=1,2, \ldots D)$

$\mathrm{o}_{m}$ : media option of $\mathrm{m}^{\text {th }}$ medium

$i_{m}:$ insertion/slot in $\mathrm{m}^{\text {th }}$ medium

$s_{c}^{d}$ : criteria ( here $s_{c}=1,2$ i.e. age and income level for all 
$d=1,2, . . . D)$ under consideration for $\mathrm{s}^{\text {th }}$ segment of $d^{\text {th }}$ demand centre

$r_{s m o_{m} s_{m}}^{d}$ : target consumer reach for an advertisement in $s^{\text {th }}$ segment of $d^{\text {th }}$ demand centre, $m^{\text {th }}$ medium, $\mathrm{o}_{m}{ }^{\text {th }}$ media option, $i_{m}{ }^{\text {th }}$ slot/insertion.

$R_{s m o_{m} s_{m}}^{d}$ : number of readers or viewers in $m^{\text {th }}$ medium, $\mathrm{o}_{m}$ ${ }^{\text {th }}$ media option, $i_{m}^{\text {th }}$ slot/insertion in $s^{\text {th }}$ segment of $d^{\text {th }}$ demand centre

$C_{s m o_{m} s_{m}}^{d}$ : advertisement cost of inserting one advertisement for the product in $m^{\text {th }}$ medium, $\mathrm{o}_{m}^{\text {th }}$ media option, $i_{m}{ }^{\text {th }}$ slot/insertion in $s^{\text {th }}$ segment of $d^{\text {th }}$ demand centre

$\operatorname{Min}_{s m o_{m} s_{m}}^{d}$ : minimum number of advertisements in different positions for the product in $m^{\text {th }}$ medium, $\mathrm{o}_{m}{ }^{\text {th }}$ media option,$i_{m}^{\text {th }}$ slot/insertion in $s^{\text {th }}$ segment of $d^{\text {th }}$ demand centre

$\operatorname{Max}_{\text {smo }_{m} s_{m}}^{d}$ : maximum number of advertisements in different positions for the product in $m^{\text {th }}$ medium, $\mathrm{o}_{m}^{\text {th }}$ media option, $i_{m}^{\text {th }}$ slot/insertion in $s^{\text {th }}$ segment of $d^{\text {th }}$ demand centre

$x_{s m o_{m} s_{m}}^{d}$ : decision variable corresponding to $m^{\text {th }}$ medium, $\mathrm{O}_{m}$ th media option, $i_{m}^{\text {th }}$ slot/insertion in $s^{\text {th }}$ segment of $d^{\text {th }}$ demand centre

$W s_{c m}^{d}$ : weight corresponding to $s_{c}{ }_{c}$ criteria and $m^{\text {th }}$ medium

$P_{s m o_{m} s_{m}}^{d}$ : percentage of people in $s^{\text {th }}$ segment for the product having $s_{c}{ }_{c}$ criteria for $m^{\text {th }}$ medium, $\mathrm{o}_{m}{ }^{\text {th }}$ media option, $i_{m}^{\text {th }}$ slot/insertion in $s^{\text {th }}$ segment of $d^{\text {th }}$ demand centre

A : advertising budget

\subsection{Deterministic Model}

The problem for finding the optimal number of advertisements to be allocated to different media sources $m$, $m=1,2, \ldots . M$ for the newly introduced FMCG product that would maximize the total advertising reach for all media can be formulated as:

$$
\begin{aligned}
& \operatorname{Max} Z_{m}=\sum_{d \in D} \sum_{s \in S} \sum_{o_{m} \in O} \sum_{i_{m} \epsilon S} r_{S m o_{m} s_{m}}^{d} x_{s m o_{m} i_{m}}^{d} \quad \forall m \in M \\
& \operatorname{Max} Z_{m}=\sum_{d \in D} \sum_{s \in S} \sum_{o_{m} \in O} \sum_{i_{m} \epsilon S}^{o r}\left(W s_{c m}^{d}\right. \\
& \left.* P_{S m o_{m} s_{m}}^{d}\right) * R_{s m o_{m} s_{m}}^{d} x_{s m o_{m} i_{m}}^{d} \\
& \forall s_{c}=1,2, d=1,2, . . D, \quad \forall s \in S, \quad \forall m \in M, \forall o_{m} \in O
\end{aligned}
$$

Subject to

$$
\begin{aligned}
& \sum_{d \epsilon D} \sum_{s \epsilon S} \sum_{o_{m} \in O} \sum_{i_{m} \epsilon I} C_{s m o_{m} s_{m}}^{d} x_{s m o_{m} i_{m}}^{d} \leq A \\
& x_{s m o_{m} i_{m}}^{d} \geq \operatorname{Min}_{s m o_{m} s_{m}}^{d} \forall s \in S, \quad \forall m \in M, \forall o_{m} \in O, \forall i_{m} \in I \\
& x_{s m o_{m} i_{m}}^{d} \leq \operatorname{Max}_{s m o_{m} s_{m}}^{d} \forall s \in S, \forall m \in M, \forall o_{m} \in O, \forall i_{m} \in I \\
& x_{s m o_{m} i_{m}}^{d} \geq 0
\end{aligned}
$$

$$
\begin{array}{r}
\forall s \in S, \forall m \in M, \forall o_{m} \in O, \forall i_{m} \in I \\
x_{\text {smo }_{m} i_{m}}^{d} \text { are integers }
\end{array}
$$

\subsection{Stochastic model with deterministic equivalents}

Applying chance constraint approach given by [17-19] the deterministic model given in equation (1) to equation (6) is converted to its stochastic version described from equation (7) to equation (12) as follows:

$$
=\sum_{d \in D} \sum_{S \in S}^{\operatorname{Max}} \sum_{o_{m} \in O} \sum_{i_{m} \in S} r_{s m o_{m} s_{m}}^{d} x_{s m o_{m} i_{m}}^{d} \quad \forall m \in M
$$

Subject to

$$
\sum_{d \in D} \sum_{s \in S} \sum_{o_{m} \in O} \sum_{i_{m} \in I} C_{S m o_{m} s_{m}}^{d} x_{s m o_{m} i_{m}}^{d} \leq A
$$

$$
\begin{aligned}
& x_{s m o_{m} i_{m}}^{d} \geq \operatorname{Min}_{s m o_{m} s_{m}}^{d} \forall s \in S, \forall m \in M, \forall o_{m} \epsilon O, \forall i_{m} \in I \\
& x_{s m o_{m} i_{m}}^{d} \leq \operatorname{Max}_{s m o_{m} s_{m}}^{d} \forall s \in S, \forall m \in M, \forall o_{m} \in O, \forall i_{m} \in I \\
& x_{s m o, i}^{d} \geq 0 \quad \forall s \in S, \quad \forall m \in M, \forall D_{m} \in O, \forall i_{m}
\end{aligned}
$$

Where

$$
\begin{aligned}
& \sum_{d \epsilon D} \sum_{s \epsilon S} \sum_{o_{m} \in O} \sum_{i_{m} \epsilon S}\left(W s_{c m}^{d} *\right. \\
& P_{S m o_{m} s_{m}}^{d} * R_{s m o_{m} s_{m}}^{d} x_{s m o_{m} i_{m}}^{d} \geq F_{A^{m}}^{-1}\left(\alpha^{m}\right) \quad \forall m \epsilon M
\end{aligned}
$$

\section{- Deriving advertising reach uncertainty}

To determine the deterministic equivalent of the reach objective the following procedure is adopted. The actual advertising reach may differ from the planned advertising reach due to customer behavior , market competition , choice of media etc. Hence, advertising reach is probabilistic in real practice. Here, advertising reach is assumed to be probabilistic with known mean and standard deviation. The mean and standard deviation data is based on historical evidences. Let $A^{1}$ be the aspired advertising reach for recyclable print media objective function and $A^{2}$ be the aspired advertising reach for the website media objective function. This aspiration level may be obtained by getting the ideal solution of solving both the print media and website media objectives separately. Applying chance constraint approach the probabilistic equations for advertising reach objective functions are written as :

$$
\begin{gathered}
P\left(\sum_{d \epsilon D} \sum_{s \epsilon S} \sum_{o_{m} \in O} \sum_{i_{m} \in S} r_{s m o_{m} s_{m}}^{d} x_{s m o_{m} i_{m}}^{d} \geq A^{m}\right) \geq \\
\alpha^{m}, \quad \forall m \in M
\end{gathered}
$$

$$
F_{A^{m}}\left(\sum_{d \in D} \sum_{s \in S} \sum_{o_{m} \in O} \sum_{i_{m} \in S} r_{s m o_{m} s_{m}}^{d} x_{s m o_{m} i_{m}}^{d}\right) \geq \alpha^{m}
$$

Which implies

$$
\begin{aligned}
& \sum_{d \in D} \sum_{S \in S} \sum_{o_{m} \in O} \sum_{i_{m} \in S} r_{s m o_{m} s_{m}}^{d} x_{s m o_{m} i_{m}}^{d} \\
& \geq F_{A^{m}}^{-1}\left(\alpha^{m}\right) \quad \forall m \in M
\end{aligned}
$$


Deterministic equivalent in inequality equation (1.15) preserves the linearity of inequality equation (14).

\subsection{Goal programming approach}

The problem formulated in section 4.4 can be rewritten as

$$
\operatorname{Max} Z_{m}=\sum_{d \in D} \sum_{s \in S} \sum_{o_{m} \in O} \sum_{i_{m} \in S} r_{s m o_{m} s_{m}}^{d} x_{s m o_{m} i_{m}}^{d} \quad \forall m \in M
$$

Subject to

$$
\sum_{d \in D} \sum_{S \in S} \sum_{o_{m} \in O} \sum_{i_{m} \in I} C_{s m o_{m} s_{m}}^{d} x_{\text {smo }_{m} i_{m}}^{d} \leq A
$$

$$
\sum_{d \epsilon D} \sum_{s \in S} \sum_{o_{m} \in O} \sum_{i_{m} \epsilon S} r_{s m o_{m} s_{m}}^{d} x_{s m o_{m} i_{m}}^{d} \geq F_{A^{m}}^{-1}\left(\alpha^{m}\right)
$$

$$
\begin{gathered}
x_{s m o_{m} i_{m}}^{d} \leq \operatorname{Max}_{s m o_{m} s_{m}}^{d} \forall s \in S, \forall m \in M, \forall o_{m} \in O, \forall i_{m} \in I \\
x_{s m o_{m} i_{m}}^{d} \geq 0 \quad \forall s \epsilon S, \forall m \epsilon M, \forall o_{m} \in O, \forall i_{m} \in I \\
x_{s m o_{m} i_{m}}^{d} \text { are integers }
\end{gathered}
$$

This problem when solved using standard mathematical programming approach provides infeasible solution. In order to obtain compromise solution, goal programming approach is used to solve the problem defined from equation (1.16) to equation (1.22) in two stages .

\section{Stage 1}

Minimize

$$
\begin{aligned}
g_{0}(\gamma, \delta, X)=\delta^{d}+ & \sum_{d \in D} \sum_{s \in S} \sum_{o_{m} \in O} \sum_{i_{m} \in I} \gamma_{s m o_{m} i_{m}}^{d} \\
& +\sum_{d \in D} \sum_{S \in S} \sum_{o_{m} \in O} \sum_{i_{m} \in I} \delta_{s m o_{m} i_{m}}^{\prime d}
\end{aligned}
$$

Subject to

$$
\begin{aligned}
& \sum_{d \in D} \sum_{S \in S} \sum_{o_{m} \in O} \sum_{i_{m} \in I} C_{s m o_{m} s_{m}}^{d} x_{s m o_{m} i_{m}}^{d}+\gamma^{d}-\delta^{d}=A \\
& x_{s m o_{m} i_{m}}^{d}+\gamma_{s m o_{m} i_{m}}^{d}-\delta_{s m o_{m} i_{m}}^{d} \\
& =0 \quad \forall s \in S, \forall m \in M, \forall o_{m} \in O, \forall i_{m} \in I \\
& \begin{array}{c}
x_{s m o_{m} i_{m}}^{d}+\gamma_{s m o_{m} i_{m}}^{\prime d}-\delta_{{ }_{s m o_{m} i_{m}}^{\prime}}^{d}=0 \quad \forall s \epsilon S, \forall m \epsilon M, \\
\forall o_{m} \epsilon O, \forall i_{m} \in I
\end{array} \\
& x_{s m o_{m} i_{m}}^{d} \geq 0 \quad \forall s \in S, \quad \forall m \in M, \quad \forall o_{m} \in O, \quad \forall i_{m} \in I
\end{aligned}
$$

$$
x_{s m o_{m} i_{m}}^{d} \text { are integers }
$$

Let $\left(\gamma^{0}, \delta^{0}, X^{0}\right)$ be the optimal solution for the problem equation (1.23) to equation (1.28). Where $\gamma_{s m o_{m} i_{m}}^{d}$ and $\delta_{s m o_{m} i_{m}}^{d}$ are the over and under-achievement (negative and positive deviational) variables of the goals for their respective objective/constraint function for lower bound constraints and $\gamma_{s m o_{m} i_{m}}^{\prime d}$ and $\delta^{\prime d}{ }_{{ }^{\prime} o_{m} i_{m}}$ are the over and under-achievement (negative and positive deviational) variables of the goals for their respective objective /constraint function of upper bound constraints.

Stage 2

Minimize

$$
g(\gamma, \delta, X)=\sum_{m=1}^{M} \varphi_{m} \gamma_{m}
$$

Subject to

$$
\begin{aligned}
& \sum_{d \epsilon D} \sum_{s \epsilon S} \sum_{o_{m} \in O} \sum_{i_{m} \epsilon S} r_{s m o_{m} s_{m}}^{d} x_{s m o_{m} i_{m}}^{d}+\gamma_{m}-\delta_{m}= \\
& F_{A^{m}}^{-1}\left(\alpha^{m}\right), \forall m \epsilon M \\
& g(\gamma, \delta, X)=g_{0}\left(\gamma^{0}, \delta^{0}, X^{0}\right)
\end{aligned}
$$

Along with constraints from (1.24)-(1.28)

Here $\varphi_{m}$ denotes $\mathrm{m}^{\text {th }}$ objective function weights. $\gamma_{m}$ and $\delta_{m}$ denotes the positive and negative deviational variables respectively corresponding to the $\mathrm{m}^{\text {th }}$ objective function ( for $\mathrm{m}=1,2 \ldots \mathrm{M})$.

\section{FUTURE SCOPE}

Present research can be improved by incorporating some qualitative issues such as customer loyalty, price variations among media service providers etc. In addition, the problem can further be considered to prevail in a time dependent dynamic environment.

\section{REFERENCES}

[1] M Nouwen , K U Leuven , B Leuven (2018). Towards sustainable media practices in families with young children. Proceedings of $\mathrm{CHI}$ conference on human factors in computing systems. ISBN 978-1-4503-5621-3.

[2] Jones , G.E. , Plassmann, K. , York ,E.H. , Hounsome B. Jones, D.L., Canals, L.M.I . 2009. Vulnerability of exporting nations to the development of a carbon label in the United Kingdom. Environmental Science. 12 (4): 479-490.

[3] De Kluyver(1978) Hard and soft constraints in media scheduling. Journal of Advertisement Research. 18: 27 31.

[4] Jha, P.C., Aggarwal ,R. , Gupta ,A. and Aggarwal ,S. 2012. Optimal advertising Media selection model for promotion of Multi Products Segmented Market. Journal of Statistics \& Management Systems (JSMS). Taru publications. 15(1): 61-80.

[5] Saluja,D. and Saluja, M.S. 2011. A Sustainable Growth of Market: Green Marketing. Journal of Business \& Information Management . 4:1-17.

[6] B Contini, B. 1968. A stochastic approach to goal programming. Operations Research. 16 (3): 576-586.

[7] Simula,H. , Lehtimäki,T. Salo, J. 2009. Managing greenness in technology marketing. Journal of Systems and Information Technology. 11(4): 331-346. 
[8] Rademaker, C.A. 2013. Green media : Exploring Green Media Selection and its impact on communication effectiveness. Doctoral thesis submitted to Department of Marketing and Strategy at the Stockholm School of Economics (SSE). ISBN 978-91-7258-878-3.

[9] Reichart, I. and Hischie, R. 2003. The Environmental Impact of Getting the News A Comparison of On-Line, Television, and Newspaper Information Delivery. Journal of Industrial Ecology. 6 (3-4): 185-200.

[10] Jha, P.C. ,Aggarwal ,S., Gupta, A. and Aggarwal , R. 2016. Multi-criteria media mix decision model for advertising a single product with segment specific and mass media. Journal of Industrial and Management Optimization . 12(4):1367-1389.

[11] Jha, P.C. ,Aggarwal ,R., Gupta, A. 2011. Optimal media planning for multi-product in segmented market. Applied Mathematics and Computation. 217 (16): 68026818, Elsevier Science Direct.

[12] Bhattacharya, U.K. 2010. A chance constraints goal programming model for the advertising planning problem. European Journal of Operational Research. 192 : 382-395.
[13] Edwardsson, M.P. 2014. Environmental aspects of media scenarios for the future ICT society - a qualitative study. Proceedings of the 2014 conference ICT for Sustainability, Stockholm, Atlantis Press. ISBN (online): 978-94-62520-22-6

[14] Edwardsson, M.P. 2014. Explorative scenarios of emerging media trends", Journal of Print and Media Technology Research. 3: 195-206.

[15] Bansal ,P. Roth, K. 2000. Why companies go green: a model of ecological responsiveness. Academy of Management Journal. 43 (4) : 717-736.

[16] Dahlén, M. Friberg,L. , Nilsson, E. 2009. Long Live Creative Media Choice. Journal of Advertising. 38 (2): 121-129.

[17] Charnes, A. , Cooper, W. , DeVoe, J.K. , Learner, D.B. and Reinecke, W. 1968. A goal programming model for media planning. Management Science. 14: 422-430.

[18] Charnes, A. and Cooper , W. 1959. Chance-constrained programming. Management Science, 5: 73-79.

[19] Charnes, A. and Cooper , W. 1963. Deterministic equivalents for optimizing and satisfying under chance constraints", Operations Research , 11: 18-39. 